\title{
Research on Mongolian Translation of Terminologies for New Media
}

\author{
Sarul $^{*}$ \\ School of Mongolian Studies, Inner Mongolia University, Hohhot \\ *Corresponding author
}

\begin{abstract}
With the increasing development of the new media, the new terminologies related to the publication industries are sprouting, bringing about the critical issue on how to formulate and implement them in Mongolian. At present, there are trends that the translators adopt the new terminologies according to their own understandings, therefore, the terminologies of Mongolian characteristics are converted based on her lexicology as well as the transliteration of the foreign words in terms of its close approximation in Mongolian phonetic sound when it comes to the terminologies on the new media in society. However, the coexistence of different terms where two or more terminologies on the same phenomenon results in the inconsistency should be normalized and unified as well. This paper, based on the current situation where the existing translation of the terminology involving the new media is not standardized, puts forward the view that when introducing the new terminologies, we should analyze the objective reasons and stick to the principle that we not only make full use of the rich resources in the Mongolian lexicology, giving new meanings to the old words or creating new terms but also should we transliterate the foreign words if there are not corresponding counterparts in Mongolian regardless the fact that the arising terminologies on new media are novel concept yet constantly developing and the coexistence of different terminologies is on their way to the standardization.
\end{abstract}

Keywords: New media, Terminology, Translation

\section{INTRODUCTION}

First of all, as is known to all that the new media is the organic product of the computer technology, the Internet technology and the informatics, which are so advanced that all the nations and countries in the world have entered the new media era. With the development of the new media, many new problems related to it have emerged. Among them, the issue of the terminology translation involving new media is becoming an urgent issue. Because the main function of the new media is spreading news to every household, the first problem encountered in the popularization process is how to be expressed correctly in the language of the people and how it will be introduced to the people for the sake of their familiarity and popularization.

It is proved that when something new is born, it is not uncommon for it to be followed with multiple names. During the application process, after a certain period of time, some of the new terms are often recognized by the general public and some are eliminated automatically. For example, computers are translated into "electronic machine" or "electronic brain" and the mobile phones are translated as "pocket phones". At present, those terms on the new media are out of use and the acknowledged words "computer" and "mobile phone" are handed down to the general public of Mongolians. Right now, we are in the early stages of the application where the terms involved in the new media are not standardized and consistent, for the different names are being used in all sectors of society. We believe that the issues involving the unification, standardization and popularization of the terminologies on the new media play important roles in the development of the Mongolian new media itself, the development of the Mongolian language and the script, and even it is of great significance to the expansion and spreading of the Mongolian culture.

\section{PRESENT SITUATION OF MONGOLIAN NEW MEDIA TERMINOLOGIES}

Secondly, with the development of the times, the Mongolian new media started from the mobile news and developed into a variety of the new media via mobile phones. In this development process, in terms of terminology involving the new media, the media headed by Inner Mongolia Daily attaches a great importance to the terminology issues, setting up a special working group to formulate and implement the new words or the terminologies. But the terms involved in the new media are not standardized and consistent, which not only affects the quality of the media reports but also brings the language barriers to the practical application in all walks of life and even to the cultural exchanges between different countries and ethnic groups. We observe the following two conditions in the use of the new media terminology. 
(1) On the one hand, different translations are evident in the publishing and the academia. When observing the use of terminologies involved in the new media, it is more common that the same thing or the phenomenon is translated in one way by the media and in the other way by colleges and universities. For example, while the media employs the free translation, using Mongolian terms such as "suljiye for Site, jirgiye for Wechat, burge for Podcast, biqil burge for Blog, siljiye for App, qooriya for Tik Tok, qergiye for Kuaishou, one of the social media apps", the academic circles directly adopt the literal translation from the foreign language, using the foreign words such as "Site, Wechat, Blog and Podcast".

The above examples show that the media creates new words on the basis of the Mongolian lexicology features or makes meaningful expressions out of the original vocabulary of Mongolian language while the academic circles directly transliterate the new terminologies from English. But what needs to be explained here is that the media and the academia are not in the same page when it comes to the terminologies on the new media thus relatively resulting in the following scenarios.

From the perspective of the translation studies, both the free translation and the literal translation are reasonable. However, the same terminology is translated variously because of different translators. Although the reasons of this phenomenon come in the subjective and the objective aspects, the main reason is directly related to the subjective knowledge as well as understanding of the translators and are also directly related to the receiver themselves in the process of their transmission.

(2) On the other hand, the same terminology is used different internally. In the same unit or the unit of the same kind, although the terms are used relatively consistent, in many cases, the same unit or the same office has different usages for the same terms. For instance, in the Inner Mongolia Daily, there comes the following terminologies for the same matter: WeChat (2014.1.8), Blog (2014.4.9), Wechat account (2016.8.1), Website (2014.2.25), Internet (2015.12.31), Big Data (2015.12.31), Big Data Base (2016.1.14), Video (2016.7.11), Visual (2016.7.11); Internet of Things (2015.2.2), Internet of Commodities (2015.2.9), Internet of Circulate (2015.11.23), etc. Meanwhile, in the thesis of Inner Mongolia University, there appear different terminologies as WeChat platform, WeChat public platform, WeChat public number and WeChat public account and so on. That is to say, the same terminology in the same newspaper has different references and applications. In addition, the same graduation thesis of the same university has different names for the same terminology. Especially in the same daily newspaper, there is a different representation for the same terminology or in an academic paper, there is a different reference to the same terminology. Due to the absence of a reference dictionary for using a new terminology, people translate the terminology of the new media based on their own knowledge. After the emergence of a new thing, although it is inevitable that there are different ways of naming a terminology in the process of its maturity, it is taken for granted that the terminology must be consistent in a piece of newspaper, a magazine and a book. However, our research shows that the terminology involved in the new media is not only inconsistent in abroad but also at home. Especially in a daily newspaper or in an article, the different use of the same terminology is due to the irresponsible and careless attitudes of the people involved.

\section{METHODS OF STANDARDIZING NEW MEDIA TERMS}

There are certain rules and methods for translating a new terminology from one language to another. That is, from the perspective of the translation methods, there include free translation, literal translation, synthetic translation and extended translation. From the perspective of the principles, the translation should be based on the lexicology of the language, creating new words based on the existing vocabulary as well as introducing foreign languages. At present, the Mongolian translation of the new media terms commonly used today shows that though the process of the terminology being incorporated into the mother tongue is inconsistent and non-standard, it conforms to the specific method of the terminology translation. For instance, methodologically the terminology of "Wechat" is a transliteration while principally it is also a loan word from the foreign language. Then the word "Internet" is a terminology of literal translation in method and it is also a new terminology created based on the meta-language in Mongolian according to the translation principle. Judging from the translation of terminologies on the new media, we will not encounter the situations where translation methods are not applicable. However, in the inconsistent and irregular current state, opinions vary on whether to use the terminology in Mongolian language to express or to introduce directly from a foreign language by using literal translation, which brings the problem that the same terminology on the same new media is used differently in the society.

When a new terminology is exposed, it will be first applied to and disseminated to various media reports. In the process of translating the specialized terminology, the authoritative media organizations as well as the research institutes have different versions due to the different perspectives and positions of the translators, which has a varying degree of spreading because of the different opinions of the receivers. Since there is no reference dictionary on the terminology related to the new media, people will mainly consult to the social use.

Therefore, those terminologies on the new media such as "Suljiye for internet, Siljiye for App, Jirgiye for Wechat, Qargiye for Kuaishou" have come into being, which is based on the original vocabulary of Mongolian language. At the same time, there are some of the terminologies on the new media that are transliterated from the foreign words such as "Wechat, Blog, and Client". In addition, there are also some of the semi-free translated and semi-transliterated terminologies as "Big data, podcast, 
and video networks". But most importantly, its standardization and the popularization during the use of the terms are more significant than when two or even more terminologies on the same new media are being referred to at first. Therefore, people also have different views on the terminologies on the new media. Some people hold the view that we try to use the mother tongue lexicology as a support when determining new terms, and explore the old words for their new meanings or create new words while others believe that the names of the new media do not have to be translated into Mongolian to express but rather to translate directly from the foreign languages.

Then we come to the dilemma on how to appropriately translate the new media terminologies into Mongolian. We believe that the coexistence of different terms within a certain period of time after the new term was created is a general law in the terminology specification process. It is not necessary to argue which is right and which is wrong because it is common that the use of different names for the same thing leads to the one cognized by the public in the process of the usage in order to achieve the specification of the term and integrate into the mother tongue. For example, when the mobile phone was first used, the Mongolians translated it into "pocket phone" or "hand phone". Now, they only use the name "mobile phone". Moreover, computers were named "electronic brains" or "electric brains" and so on. But now the name of "computer" is used. As a result, on the issue of the new media terminology, we should maintain an optimistic attitude and we should also accept the reality where there are different terminologies can be coexisted at the same time. But this is not an issue that anyone can be able to use the new terms randomly but it is used by refining and compressing the new terms in various aspects of society rather than acknowledging different terms side by side on any personal will. Another issue that needs attention is that the authoritative units would selectively use the current terminologies rather than unscientifically to copy their terminologies because of the social status or the social influence of the units. Regarding how the terminologies involved in the new media are being used, we believe that first of all, when the meaning of the event can be accurately expressed and according to the original word in their mother tongue, we should use the old words with new meanings or create new words, which is not only consistent with the principles of translating terminologies but also is useful to the regularity of terminologies as well as the enrichment of the mother tongue vocabulary. However, in the case where the terminology at hand cannot be accurately interrupted at the time and it is also difficult to compose new words to express the source text, therefore it is realistic to directly transliterate the foreign terminologies for the new media. As far as I am concerned, we will not accept the extreme views that in the translation of the new media terms, we must express them in Mongolian or transliterate the new terminologies from a foreign language. It has been found that this is not only inconsistent with the rule of the terminology use but also with the law of the language development. For example, the use and the specification of a series of terminologies such as "computer, mobile phone, Internet, social network, virus, netizen, video ..." lay a solid foundation for our view. In our opinion, the following countermeasures should be taken on the terminologies involved in the new media:

1. Relevant units and institutes of higher education should strengthen scientific research cooperation to promote the unification of terminologies. The advent of the new media terminology first involves with the media units and the institutes of higher education. The popularization of the terminology related to the new media plays an important role in the media and in the fields of the education and the academia. For the introduction of the terminologies related to the new media, the media and the academia should cooperate to determine the most authoritative terminology that can be accepted by all sectors of society and should be undergone the supervision of general public in the application process, paying attention to the feedback information and improving the use of the new terminology in time.

2. Relevant departments should formulate and implement new terminologies in all areas of society, accept the supervision from all walks of life, revise them in time and promptly promote the standardization of terminologies. In this regard, the "Mongolian Terminology Committee on the Mongolian Language Coordination from the eight provinces" and the "Mongolian Terminology Committee in Inner Mongolia Autonomous Region" specify and implement the new terminologies in the form of an "official documents", which is of great significance to the formulation, implementation and standardization of the new terminologies.

3. Publish a dictionary of terminologies and build a corpus of terminologies. The terminology dictionary is a manual for translating terminologies. In China, there are many Chinese terminology dictionaries. These dictionaries not only have comprehensive contents covering various disciplines and vocations but also have specially designed dictionaries related to a certain subject and a certain profession. However, dictionaries of Mongolian terminologies are extremely rare, especially those related to the subject of publishing and journalism and communication fields almost never existed. Therefore, it is important to compile and print a dictionary of terminology which can also be codified into other dictionaries and become a part of them so as to give references to the users. In addition, terminology databases can be established to lay the foundation for the establishment of terminology dictionaries and to provide services to users. Moreover, we should build a corpus of terminologies that could build up a good basis for the advancement of the dictionary of terminology for the public to consult to in the future. Compared with compiling a dictionary, there are some advantages for creating corpus because with the development of science and technology and with the growing number of the new terminologies, the database of terminologies can be expanded and updated at any time and the users can look up at any time. However, it should be noted that we must strengthen the selection of the fine terminology in the database to ensure the authoritative features of the terminology in the database. On the basis of 
establishing a professional or comprehensive terminology database, an electronic dictionary of the terminology can also be developed for users to take advantage of.

\section{CONCLUSION}

All in all, there are two cases concerning with the terminology translation involving the new media, namely the translation based on the mother tongue of Mongolian and the direct transliteration from the foreign language. Unfortunately, various walks of life hold different views on the terminology. Besides, the same unit has different usages for terminologies as well. Opinions vary on whether to translate the terminology on the basis of the native Mongolian language or to transliterate it directly from the foreign language. We believe that with the development of the new media and with the new terms constantly being produced, first of all, although we should base on the mother tongue, focusing on the use of the specified terminologies, we must pay attention to the appropriate semantic meaning of the terminologies. Under the condition where the terminology proper will not be represented by the current vocabulary in the mother tongue at all, according to the existing vocabulary in the mother tongue, a new word can be created to match the semantic meaning of the new thing or you can directly transliterate the noun from a foreign language to quote the new terminology when there is nothing in common with the native language. In the application process, after the supervision of various circles of society, some of the translation of terminologies is integrated into the mother tongue within a certain period of time so that the standardization of the terminology is automatically achieved. But the formulation, implementation, and standardization of terms do not only depend on the casual development, but also the organized activities must be required. For example, the media and the academic community of the higher education should strengthen their cooperation, discuss and decide on the new terminologies, and implement them uniformly. The terminology committee organizes scholars from all walks of life in the society in order to formulate, formally release and organize them from various departments. Through the compilation of the dictionary of the terminology as well as the creation of the terminology corpus, the terminologies related to the new media and the formulation, implementation and promotion of all the other new terminologies can also be promoted.

\section{ACKNOWLEDGEMENT}

This work is partially supported by National Natural Science Foundation Project (the project No. is 61662050).

\section{REFERENCES}

[1] Mongolian Terminology Committee on the Mongolian Language Coordination from the eight provinces" and the "Mongolian Terminology Committee in Inner Mongolia Autonomous Region, "Official Documents of Mongolian Terminology" (No. 130-132).

[2] Horina, "Study on the new words in the new era Inner Mongolian Daily News", Master's thesis of Inner Mongolia University, 2018.

[3] D. Bater, "On the Mongolian Terminology", Nationality Publishing House, 2008.

[4] Somburbatu, "Neologisms Dictionary of Mongolian and Chinese", Nationality Publishing House, 2007.

[5] Mongolian terminology committee on the Mongolian terminology in Inner Mongolia Autonomous Region, "Papers on Mongolian Terminologies", Inner Mongolian Science and Technology Press, 1999.

[6] L. Bater, "An analysis of the way to produce contemporary Mongolian words and expressions", Journal of Inner Mongolia Normal University, 2014 (2).

[7] L. Bater, "On the new words and expressions of contemporary Mongolian”, Journal of Inner Mongolia Normal University, 2015(2).

[8] Dagula, "On the translation of the new words of Mongolian news in new era", Mongolian Studies in China, 2016(3).

[9] Dagula, "On the application of new words and media in Mongolian news", Social Science of Inner Mongolia, 2016(3) 